\title{
Characterizing API Elements in Software Documentation with Vector Representation
}

\author{
Thanh Van Nguyen \\ ECpE Department \\ lowa State University, USA \\ thanhng@iastate.edu
}

\author{
Anh Tuan Nguyen \\ ECpE Department \\ lowa State University, USA \\ anhnt@iastate.edu
}

\author{
Tien N. Nguyen \\ ECpE Department \\ lowa State University, USA \\ tien@iastate.edu
}

\section{Categories and Subject Descriptors}

I.2.7 [Natural Language Processing]: Text Analysis

\section{Keywords}

Software Documentation, Word2Vec, Software Libraries

\section{RESEARCH PROBLEM}

In software engineering (SE), documentation for developers (e.g., developers' guide, API documentation), users' documentation, informal documentation [7, 13] (e.g., developers' forums, mailing lists, development communities' discussions, etc.), and issue reports are of much interest for software engineers. This so-called software documentation is a crucial resource for them in understanding various aspects of a software development process.

A unique nature of such documentation is the presence of code elements embedded in natural language texts that explain their purposes, usages and mutual connections with others. It is desirable to exploit this nature to some extent for effectively leveraging information on libraries/frameworks. In fact, we have witnessed successful work in exploring such data to support a variety of SE applications. These include summarizing classes and methods [7], enhancing IDEs with code search [3, 14], API code search [2, 6, 11, 16], application searching [10], finding recurring bug fixes [5], extracting information from developers' emails, issue reports [15], etc.

Some recent work $[7,13]$ has explored the mixture of code and text in software documentation for different purposes. While Rigby et al. [13] proposed a method to discover code elements contained in documents (i.e., embedded APIs hereafter), Guerrouj et al. [7] aimed to generate summaries for annotated code elements based on the words frequently preceding them. However, none of the aforementioned approaches is capable of further capturing semantic relations of embedded APIs with related words and with other relevant APIs at the same time. It is hence natural to find representations of embedded APIs and words in software documentation to achieve that goal. The representations should allow a high level of abstraction and preserve linguistic regularities among words and APIs. Once the goal is achieved, the representations would facilitate many SE applications where the connections between APIs and texts are needed.

Permission to make digital or hard copies of part or all of this work for personal or classroom use is granted without fee provided that copies are not made or distributed for profit or commercial advantage and that copies bear this notice and the full citation on the first page. Copyrights for third-party components of this work must be honored. For all other uses, contact the owner/author(s).

ICSE '16 Companion, May 14 - 22, 2016, Austin, TX,USA

(C) 2016 Copyright held by the owner/author(s).

ACM ISBN 978-1-4503-4205-6/16/05 . .\$15.00

DOI: http://dx.doi.org/10.1145/2889160.2892658
Example applications include seeking APIs with same functionality, retrieving API usages given a textual query, recommending related APIs for programming tasks, and so on. Importantly, with such representations, a variety of SE tasks should no longer rely heavily on parallel corpora of code and descriptions.

\section{APPROACH AND UNIQUENESS}

In this paper, we conjecture that API elements and words being semantically relevant often occur in similar contexts and therefore should have similar representations. We hence consider an embedded API element as a word and aim to characterize this API via a low-dimensional continuous vector that encodes information of many contexts in which that API appears as possible. This vector representation can be learned from a large corpus of software documentation as it typically contains natural language texts that describe the functionality of an API, its usages and relationships with other related ones. Let us show an excerpt of the FilelnputStream's Javadoc: "A FilelnputStream obtains input bytes from a file in a file system. FilelnputStream is meant for reading streams of raw bytes such as image data... [A constructor] creates a FilelnputStream by opening a connection to an actual file, the file named by the File object [file] in the file system. A new FileDescriptor object is created to represent this file connection." As seen, the class FilelnputStream is closely related to "read", "input", "file" because they are described in similar contexts such as "obtains input bytes from file", "reading streams of bytes". Meanwhile, "opening connection to file..." reveals the relation between FilelnputStream and File. This example suggests that by observing as many contexts of surrounding APIs and words, we could reveal the semantic relations among them.

Inspired by recent successes in learning word representation in natural language processing, we choose to use Word2 Vec model [12] to characterize API elements (as well as words) in a low-dimensional semantic space. Word2 Vec is trained to both maximize the likelihood of seeing a word given its surrounding contexts and minimize training time. In addition to a very fast training speed, Word2 Vec model can preserve linguistic regularities among words in two ways. First, the words being used in the similar contexts tend to have similar embeddings in the continuous vector space [4]. Second, several semantic relations among words can be captured via simple vector operations [12]. For example, V("Italy")-V("Rome") $\approx V$ ("France")-V("Paris"), where $V$ denotes Word2Vec vector and the minus sign denotes vector subtraction operation. Thus, given "Italy", "Rome", and "France", one can retrieve "Paris". Our empirical study (Section 3) confirms that these properties still hold for the vector representations learned from a large corpus of documentation. That is, the APIs with similar functionalities have similar embeddings; and the APIs and related words are close to each other in the vector space without explicit word matching. 


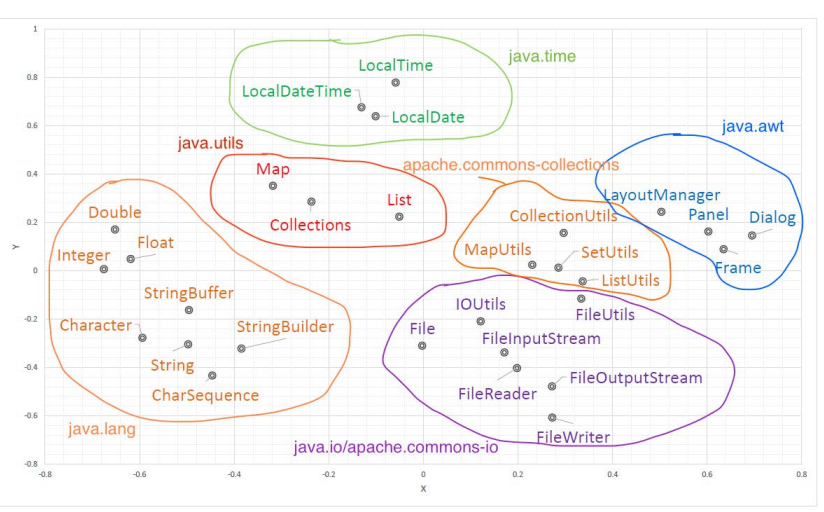

Figure 1: 2-D PCA projection of some JDK and Apache API vectors

\section{EXPERIMENTAL RESULTS}

We conducted experiments to study the Word2Vec representations for English words and embedded APIs in software documentation.

Data Preparation. To train Word2Vec model (CBOW architecture [12] was used), we used two documentation corpora from the JDK and Apache documentation $[9,1]$. The dataset contains the descriptions of 42,934 APIs in which embedded elements are annotated.

In addition, a validation dataset was obtained from [8] to evaluate the learned model for the application of API usage code search (See Section 3.2). This set includes 90 pairs of which each has an English query and its corresponding code fragment (See Table 2).

\subsection{Feature Vector Space}

In this experiment, we observed the characteristics of the learned vectors for words and embedded API elements. We first selected some common APIs and projected their corresponding feature vectors into a two-dimensional space to observe the geometric arrangements among them. Figure 1 shows a PCA visualization of the vectors. The vector space is separately divided into six clusters that correspond to six different packages. For example, String, StringBuffer and StringBuilder in the package java.lang have very similar embeddings since they often appear together and are similarly described in the Java documentation. Interestingly, API elements for file manipulation such as IOUtils and FileUtils in Apache can be grouped in the same cluster as File and FilelnputStream in $J D K$. This observation indicates that the representation learned on documentations is capable of capturing similar contexts surrounding similar APIs to reveal the semantic relations among them. This also suggests us to use this approach to map API elements with similar functions across different libraries or programming languages due to the similar nature of their descriptions in corresponding English documentations.

In the next experiment, we studied the relationship between English words and related API elements in this representation. To ensure objectivity, we selected to use the English queries and API elements from the validation dataset, which is different from the training data. Specifically, we chose four queries with different topics in [8]. We projected the vectors of individual words in each query and those of API elements in corresponding API sequences to the PCA visualization (Figure 2). As seen, the result shows the Word2Vec representation is able to capture semantic relations between API elements and English words without explicit textual matching. For instance, terms like "copy" and "file" are closer to File.File, Files.copy and FileUtils.copyFile than to any other APIs. The insight is that the words often used to describe the API elements occur in similar contexts of those API elements. This suggests that the vector representation has promising potential for API element

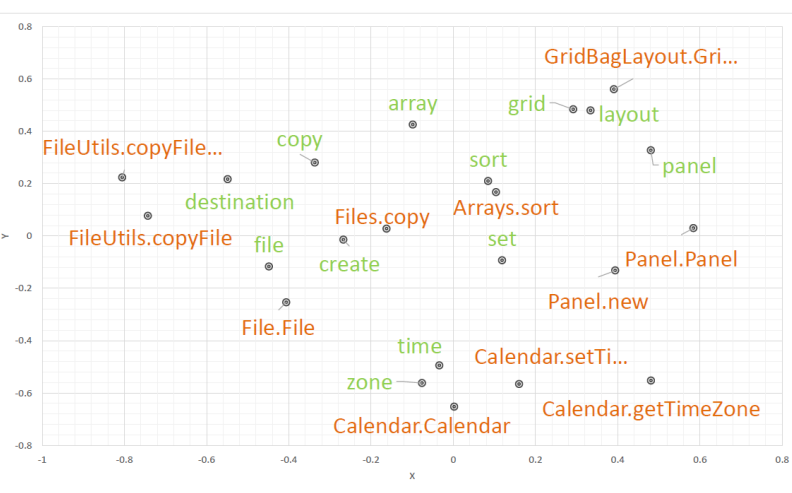

Figure 2: 2-D PCA projection of some API and word vectors

Table 1: Top-ranked Accuracy in API Example Code Search

\begin{tabular}{lllll}
\hline top-1 & top-2 & top-3 & top-4 & top-5 \\
\hline $34.6 \%$ & $48.1 \%$ & $63.5 \%$ & $75.0 \%$ & $78.8 \%$ \\
\hline
\end{tabular}

recommendation and API code search given a natural language text.

Several algebraic operations still hold for word and embedded API vectors. We performed a few operations focusing on the typical analogies in the descriptions of the API elements. For instance, $V$ ("StringBuilder.append") $-V$ ("append") $+V$ ("String.concat") results in a vector that is closest to the vector representation of the word "concatenate". This analogy describes a functional relation in which a word best explains the functionality of an API element.

\subsection{API Example Code Search}

As an immediate application of our findings, we used the Word2Vec representation for embedded API elements in an API example code search in which our goal is to retrieve a sequence of API elements given an English query. Note that, Word2 Vec provides us only the representation for individual words and API elements. We still need to have the vector representations for English phrases and API sequences. For simplicity, we used a simple bag-of-words model that averages out the vectors of the individuals in those sequences, $e . g$., the vectors of a phrase is the average vector of its individual words.

Table 2: Examples of API Example Code Search

\begin{tabular}{lll}
\hline Query & Expected API Sequence & Top \\
\hline "write file" & File.File FileUtils.writeStringToFile & 1 \\
"make file" & File.File File.createNewFile & 1 \\
"copy file to destination" & File.File File.File FileUtils.copyFile & 1 \\
"delete file" & File.File File.delete & 3 \\
\hline
\end{tabular}

Tables 1 and 2 show the result of retrieving code for the queries in the validation dataset. Despite the simple representations of text and code sequences, the retrieval performance is still reasonable. With 5 suggestions, we can correctly suggest $78.8 \%$ of the cases.

Conclusion. Our results showed that Word2Vec for software documentation can capture regularities of embedded APIs and texts. Our new approach for API code search using such vectors is promising.

\section{ACKNOWLEDGMENTS}

This work was supported in part by the US NSF grants CCF1518897, CNS-1513263, CCF-1413927, CCF-1320578, CCF-1349153, TWC-1223828, CCF-1018600, and CCLI-0737029. 


\section{REFERENCES}

[1] Apache documentation. https://httpd.apache.org/docs/.

[2] S. Bajracharya, T. Ngo, E. Linstead, Y. Dou, P. Rigor, P. Baldi, and C. Lopes. Sourcerer: A search engine for open source code supporting structure-based search. In Object-oriented Programming Systems, Languages, and Applications, OOPSLA'06, pages 681-682. ACM, 2006.

[3] J. Brandt, M. Dontcheva, M. Weskamp, and S. R. Klemmer. Example-centric programming: Integrating Web search into the development environment. In Proceedings of the SIGCHI Conference on Human Factors in Computing Systems, CHI'10, pages 513-522. ACM, 2010.

[4] L. Deng and D. Yu. Deep Learning Methods and Applications - Foundations and trends in signal processing. Microsoft Research, 7:197-387, June 2014.

[5] Q. Gao, H. Zhang, J. Wang, Y. Xiong, L. Zhang, and H. Mei. Fixing recurring crash bugs via analyzing $\mathrm{Q}$ and $\mathrm{A}$ sites. In Proceedings of the 30th IEEE/ACM International Conference on Automated Software Engineering, ASE'15. IEEE CS, 2015.

[6] M. Grechanik, C. Fu, Q. Xie, C. McMillan, D. Poshyvanyk, and C. Cumby. A search engine for finding highly relevant applications. In Proceedings of the 32nd ACM/IEEE International Conference on Software Engineering - Volume 1, ICSE' 10, pages 475-484. ACM, 2010.

[7] L. Guerrouj, D. Bourque, and P. C. Rigby. Leveraging informal documentation to summarize classes and methods in context. In Proceedings of the 37th IEEE/ACM International Conference on Software Engineering, ICSE 2015, Florence, Italy, May 16-24, 2015, Volume 2, pages 639-642. IEEE CS Press, 2015.

[8] T. Gvero and V. Kuncak. Synthesizing Java expressions from free-form queries. In Proceedings of the 2015 ACM SIGPLAN International Conference on Object-Oriented Programming, Systems, Languages, and Applications, OOPSLA'15, pages 416-432, New York, NY, USA. ACM, 2015.
[9] Java platform standard edition 7 documentation. http://docs.oracle.com/javase/7/docs/.

[10] C. McMillan, M. Grechanik, D. Poshyvanyk, Q. Xie, and C. Fu. Portfolio: Finding relevant functions and their usage. In Proceedings of the 33rd International Conference on Software Engineering, ICSE '11, pages 111-120. ACM, 2011.

[11] C. McMillan, D. Poshyvanyk, and M. Grechanik. Recommending source code examples via API call usages and documentation. In Proceedings of the 2nd International Workshop on Recommendation Systems for Software Engineering, RSSE '10, pages 21-25. ACM, 2010.

[12] T. Mikolov, I. Sutskever, K. Chen, G. S. Corrado, and J. Dean. Distributed representations of words and phrases and their compositionality. In Advances in Neural Information Processing Systems 26: 27th Annual Conference on Neural Information Processing Systems 2013 (NIPS'13)., pages 3111-3119, 2013.

[13] P. C. Rigby and M. P. Robillard. Discovering essential code elements in informal documentation. In Proceedings of the 2013 International Conference on Software Engineering, ICSE'13, pages 832-841. IEEE Press, 2013.

[14] N. Sawadsky, G. C. Murphy, and R. Jiresal. Reverb: Recommending code-related web pages. In Proceedings of the 2013 International Conference on Software Engineering, ICSE'13, pages 812-821. IEEE Press, 2013.

[15] A. D. Sorbo, S. Panichella, C. A. Visaggio, M. D. Penta, G. Canfora, and H. C. Gall. Development emails content analyzer: Intention mining in developer discussions. In Proceedings of the 30th IEEE/ACM International Conference on Automated Software Engineering, ASE'15. IEEE CS, 2015.

[16] W. Zheng, Q. Zhang, and M. Lyu. Cross-library API recommendation using Web search engines. In Proceedings of the 19th ACM SIGSOFT Symposium and the 13th European Conference on Foundations of Software Engineering, ESEC/FSE '11, pages 480-483. ACM, 2011. 